\title{
NECESSARY AND SUFFICIENT CONDITIONS ON EXISTENCE AND CONVEXITY OF SOLUTIONS FOR DIRICHLET PROBLEMS OF HESSIAN EQUATIONS ON EXTERIOR DOMAINS
}

\author{
CHONG WANG AND JIGUANG BAO \\ (Communicated by James E. Colliander)
}

\begin{abstract}
In this paper, we are concerned with the Dirichlet problems of Hessian equations on exterior domains with prescribed asymptotic behavior at infinity, and we obtain the necessary and sufficient conditions on existence and convexity of radial solutions.
\end{abstract}

\section{INTRODUCTION}

In this paper, we study the Dirichlet problems of the Hessian equations outside the $n$-dimensional unit open ball $B_{1}$

$$
\begin{aligned}
\sigma_{k}\left(\lambda\left(D^{2} u\right)\right) & =1, \quad x \in \mathbb{R}^{n} \backslash \bar{B}_{1}, \\
u & =b, \quad x \in \partial B_{1},
\end{aligned}
$$

where $b \in \mathbb{R}$ is a constant,

$$
\sigma_{k}(\lambda)=\sum_{i_{1}<\cdots<i_{k}} \lambda_{i_{1}} \cdots \lambda_{i_{k}}, k=1,2, \cdots, n
$$

is the $k$ th elementary symmetric function of $\lambda=\left(\lambda_{1}, \cdots, \lambda_{n}\right)$, and $\lambda\left(D^{2} u\right)$ are the eigenvalues of the Hessian matrix $D^{2} u$.

(11) is the Poisson equation $\Delta u=1$ if $k=1$. It is clear that the radial solution of (11), (2) is

$$
\begin{aligned}
u(x) & =b+\frac{1}{n} \int_{1}^{|x|}\left(s+\alpha s^{1-n}\right) \mathrm{d} s \\
& =\left\{\begin{array}{l}
\frac{1}{2 n}\left(|x|^{2}-1\right)+\frac{\alpha}{2-n}\left(|x|^{2-n}-1\right)+b, \quad n \geq 3, \\
\frac{1}{4}\left(|x|^{2}-1\right)+\frac{\alpha}{2} \ln |x|+b, \quad n=2,
\end{array}\right.
\end{aligned}
$$

where $\alpha \in \mathbb{R}$ is an arbitrary constant.

The Hessian equation (1) is an important class of fully nonlinear elliptic equations for $k \geq 2$, especially (11) is the Monge-Ampère equation $\operatorname{det}\left(D^{2} u\right)=1$ if $k=n$. A classical theorem of Jörgens $(n=2$ [14]), Calabi $(n \leq 5[5])$, and Pogorelov

Received by the editors August 5, 2011.

2010 Mathematics Subject Classification. Primary 35J60; Secondary 35J96.

Key words and phrases. Hessian equation, necessary and sufficient condition, existence, convexity, asymptotic behavior, radial solution, exterior Dirichlet problem. 
( $n \geq 2$ [16]) states that any classical convex solution of the Monge-Ampère equations $\operatorname{det}\left(D^{2} u\right)=1$ in $\mathbb{R}^{n}$ must be a quadratic polynomial. A simpler and more analytic proof was later given by Cheng and Yau [7. Caffarelli [1] extended the result for classical solutions to viscosity solutions. Jost and Xin [15] gave a more geometric proof. In 2003, Caffarelli and Li [2] made an extension to the JörgensCalabi-Pogorelov theorem on exterior domains.

In 2, Caffarelli and Li also discussed the Dirichlet problem on exterior domains with prescribed asymptotic behavior at infinity:

$$
\left\{\begin{aligned}
\operatorname{det}\left(D^{2} u\right) & =1, \quad x \in \mathbb{R}^{n} \backslash \bar{D}, \\
u & =\varphi(x), \quad x \in \partial D, \\
u & =\frac{1}{2} x^{\prime} A x+b \cdot x+c+O\left(|x|^{2-n}\right), \quad x \rightarrow \infty,
\end{aligned}\right.
$$

where $D$ is a smooth, bounded, strictly convex domain of $\mathbb{R}^{n}, n \geq 3, \varphi \in C^{2}(\partial D)$, $A$ is a real $n \times n$ symmetric positive definite matrix with $\operatorname{det}(A)=1, b \in \mathbb{R}^{n}, c \in \mathbb{R}$. They established the following existence theorem.

Theorem 1. There exists some constant $c_{*}$, depending only on $n, D, \varphi, b$ and $A$, such that for every $c>c_{*}$ the problem (3) has a unique solution in $C^{\infty}\left(\mathbb{R}^{n} \backslash \bar{D}\right) \cap$ $C^{0}\left(\mathbb{R}^{n} \backslash D\right)$.

In $\mathbb{R}^{2}$, similar problems were studied by Ferrer, Martínez, and Milán in [1] and [12] using complex variable methods. See also Delanoë [10].

Using the Perron method, Dai and Bao [9] get the uniqueness and existence of viscosity solutions to Hessian equations with prescribed asymptotic behavior at infinity.

There exist many excellent results on solvability of Hessian equations in interior domains. For instance, Caffarelli, Nirenberg and Spruck [4 for classical solutions, Trudinger [18 for weak solutions and Urbas [21] for viscosity solutions.

To work in the realm of elliptic equations, we have to restrict the solutions to some class of functions. Let

$$
\Gamma_{m}=\left\{\lambda \in \mathbb{R}^{n} \mid \sigma_{j}(\lambda)>0, j=1,2, \cdots, m\right\} .
$$

$\Gamma_{m}$ is a symmetric cone, that is, any permutation of $\lambda$ is in $\Gamma_{m}$ if $\lambda \in \Gamma_{m} . \Gamma_{m}$ is the half space $\left\{\lambda \in \mathbb{R}^{n} \mid \lambda_{1}+\lambda_{2}+\cdots+\lambda_{n}>0\right\}$ if $m=1$, and $\Gamma_{m}$ is the positive cone $\Gamma^{+}=\left\{\lambda \in \mathbb{R}^{n} \mid \lambda_{i}>0, i=1, \cdots, n\right\}$ if $m=n$. Following [4], we give the definition of $m$-convex functions.

Definition 1. A function $u \in C^{2}\left(\mathbb{R}^{n} \backslash \bar{B}_{1}\right)$ is called $m$-convex in $\mathbb{R}^{n} \backslash \bar{B}_{1}$ if $\lambda\left(D^{2} u\right) \in$ $\Gamma_{m}$ for all $x \in \mathbb{R}^{n} \backslash \bar{B}_{1}$.

A function is called convex if it is $n$-convex.

Motivated by the works of [2] and [9], in this paper, we investigate the exterior Dirichlet problems (11), (2) with prescribed asymptotic behavior at infinity:

$$
u(x)=\frac{a}{2}|x|^{2}+c+O\left(|x|^{2-n}\right), \quad x \rightarrow \infty,
$$

for $n \geq 3$, and

$$
u(x)=\frac{1}{2}|x|^{2}+\frac{d}{2} \ln |x|+c+O\left(|x|^{-2}\right), \quad x \rightarrow \infty,
$$


for $n=2$, where $c, d \in \mathbb{R}$,

$$
a=\left(\frac{1}{\mathrm{C}_{n}^{k}}\right)^{\frac{1}{k}}, \quad \mathrm{C}_{n}^{k}=\frac{n !}{k !(n-k) !}, \quad k \geq 2,
$$

and get the necessary and sufficient conditions on existence and convexity of radial solutions.

For simplicity, we denote for $m=1,2, \cdots, n$,

$$
\Phi_{m}:=\left\{u \in C^{1}\left(\mathbb{R}^{n} \backslash B_{1}\right) \cap C^{2}\left(\mathbb{R}^{n} \backslash \bar{B}_{1}\right) \mid u \text { is an } m \text {-convex radial function }\right\} .
$$

Our first theorem gives the necessary and sufficient conditions on the existence of $m$-convex radial solutions of (11), (2) and (4) in higher dimensions.

Theorem 2. Let $n \geq 3,2 \leq k \leq m \leq n$. Then there exists a unique function $u \in \Phi_{m}$ satisfying (10), (2), (44) if and only if $c \in[\mu(-1),+\infty)$ for $m=k$, and $c \in\left[\mu(-1), \mu\left(\frac{k}{m-k}\right)\right]$ for $m>k$, where

$$
\mu(\alpha)=b-\frac{a}{2}+a \int_{1}^{\infty} s\left[\left(1+\frac{\alpha}{s^{n}}\right)^{1 / k}-1\right] \mathrm{d} s .
$$

In the special case of radial solutions, Theorem 2 shows that there is no solution of (11), (2), (4) if $c<\mu(-1)$, and the solutions gradually weaken convexity as $c$ increases from $\mu(-1)$. So we have reason to guess that the general case (11), (2), (4) has no solution if $c$ is small enough.

From the proof of Theorem 2 we know that the general solutions in $\Phi_{m}$ of (1), (21) are

$$
u(x)=b+a \int_{1}^{|x|}\left(s^{k}+\alpha \cdot s^{k-n}\right)^{1 / k} \mathrm{~d} s, \quad \alpha \in[-1,+\infty) \text { or }\left[-1, \frac{k}{m-k}\right],
$$

which gives that the asymptotic behavior of solutions is (4), where $c \in[\mu(-1),+\infty)$ for $m=k$, and $c \in\left[\mu(-1), \mu\left(\frac{k}{m-k}\right)\right]$ for $m>k$.

For $n=k=2$, (1) becomes the Monge-Ampère equation

$$
\operatorname{det}\left(D^{2} u\right)=1, \quad x \in \mathbb{R}^{2} \backslash \bar{B}_{1} .
$$

Then, we get the following theorem.

Theorem 3. There exists a unique function $u \in \Phi_{2}$ satisfying (8), (2), (5) if and only if $d \geq-1$ and $c=\nu(d)$, where

$$
\nu(d)=b+\frac{d}{4}+\frac{d}{2} \ln 2-\frac{1}{2}\left[(1+d)^{1 / 2}+d \ln \left(1+(1+d)^{1 / 2}\right)\right] .
$$

Clearly, $\nu(+\infty)=-\infty$. Differentiating (9), we have

$$
\frac{\partial \nu}{\partial d}=\frac{1}{2} \ln \frac{2}{1+(1+d)^{1 / 2}} .
$$

It follows that $\nu(d)$ increases and decreases on $[-1,0]$ and $[0,+\infty)$, respectively. Therefore,

Actually, we have

$$
\nu(d) \leq \nu(0)=b-\frac{1}{2}, \quad d \geq-1
$$

Corollary 1. The problem (8), (2), (5) has a radial convex solution in $C^{1}\left(\mathbb{R}^{n} \backslash B_{1}\right)$ $\cap C^{2}\left(\mathbb{R}^{n} \backslash \bar{B}_{1}\right)$ if and only if $d \geq-1$ or $c \leq b-\frac{1}{2}$. 
On the other hand, the radial convex solutions of (8), (2) can be expressed as

$$
\begin{aligned}
u(x)=b & +\frac{1}{2}\left[|x|\left(|x|^{2}+d\right)^{1 / 2}+d \ln \left(|x|+\left(|x|^{2}+d\right)^{1 / 2}\right)\right] \\
& -\frac{1}{2}\left[(1+d)^{1 / 2}+d \ln \left(1+(1+d)^{1 / 2}\right)\right],
\end{aligned}
$$

and then there exist a unique $d \geq-1$ and a unique $c \leq b-\frac{1}{2}$ such that $u$ satisfies (5).

The Dirichlet problem on exterior domains of $\mathbb{R}^{2}$ was studied by Caffarelli, Li in 2] and Ferrer, Martínez, Milán in [1] and [12, respectively. But their prescribed asymptotic behavior at infinity is at most

$$
u(x)=\frac{1}{2}|x|^{2}+\frac{d}{2} \ln |x|+c+O\left(|x|^{-1}\right), \quad x \rightarrow \infty,
$$

which is weaker than (5). It would be interesting to see if the existence of (8), (2), (5) remains valid without radial symmetry assumptions.

In the following two sections, we shall prove Theorem 2 and Theorem 3 respectively.

\section{Proof of Theorem 2}

To prove Theorem 2, firstly we have several simple facts.

Lemma 1. Let $\lambda=(\beta, \gamma, \cdots, \gamma) \in \Gamma_{m}, n \geq m \geq 2$. Then $\gamma>0$.

Proof. By $\lambda \in \Gamma_{m}, m \geq 2$, we have $\sigma_{2}(\lambda)>0$. That is,

$$
C_{n-1}^{1} \beta \gamma+C_{n-1}^{2} \gamma^{2}=(n-1) \gamma\left(\beta+\frac{n-2}{2} \gamma\right)>0 .
$$

Clearly, $\gamma \neq 0$. Suppose that $\gamma<0$. Then

$$
\beta<-\frac{n-2}{2} \gamma \text {. }
$$

But, by $\sigma_{1}(\lambda)>0$, we have

$$
\beta>-(n-1) \gamma
$$

This is a contradiction. Therefore, $\gamma>0$. This completes the proof.

Lemma 2. Let $\lambda=(\beta, \gamma, \cdots, \gamma) \in \mathbb{R}^{n}$ and $\sigma_{k}(\lambda)=1,2 \leq k \leq n$. Then for $k \leq m \leq n, \lambda \in \Gamma_{m}$ if and only if

$$
0<\gamma<\gamma_{m}
$$

where

$$
\gamma_{m}=\left\{\begin{array}{l}
\frac{a}{\left(1-\frac{k}{m}\right)^{1 / k}}, \quad m>k, \\
+\infty, \quad m=k
\end{array}\right.
$$

and $a=\left(1 / \mathrm{C}_{n}^{k}\right)^{1 / k}$ is given as before.

Proof. By $\sigma_{k}(\lambda)=1$, we have

$$
C_{n-1}^{k-1} \beta \gamma^{k-1}+C_{n-1}^{k} \gamma^{k}=1 .
$$

That is,

$$
\beta=\frac{\gamma}{k}\left(n a^{k} \gamma^{-k}-n+k\right)
$$


By the definition of $\lambda \in \Gamma_{m}$, we have for $j=1,2, \cdots, m$,

$$
\begin{gathered}
\sigma_{j}(\lambda)=C_{n-1}^{j-1} \beta \gamma^{j-1}+C_{n-1}^{j} \gamma^{j}>0, \\
\gamma^{j-1}(j \beta+(n-j) \gamma)>0 .
\end{gathered}
$$

From Lemma 1, we get $\gamma>0$, and

$$
j \beta+(n-j) \gamma>0
$$

By (12), we have

$$
\begin{gathered}
j \frac{\gamma}{k}\left(n a^{k} \gamma^{-k}-n+k\right)+(n-j) \gamma>0, \\
a^{k} \gamma^{-k}>1-\frac{k}{j}, \quad j=1,2, \cdots, m,
\end{gathered}
$$

which is equivalent to

$$
a^{k} \gamma^{-k}>1-\frac{k}{m}
$$

This lemma is proved.

Lemma 3. For $2 \leq k \leq m \leq n$, assume that $u \in C^{1}\left(\mathbb{R}^{n} \backslash B_{1}\right) \cap C^{2}\left(\mathbb{R}^{n} \backslash \bar{B}_{1}\right)$ is a radial solution of (11), (2). Let

$$
\alpha:=\mathrm{C}_{n}^{k}\left(u^{\prime}(1)\right)^{k}-1 .
$$

Then $u$ is $k$-convex if and only if $\alpha \in[-1,+\infty)$, and $u$ is $m$-convex if and only if

$$
\alpha \in\left[-1, \frac{k}{m-k}\right], \quad m=k+1, \cdots, n .
$$

Proof. Assume that

$$
u(x)=u(r)=u(|x|) \in C^{1}\left(\mathbb{R}^{n} \backslash B_{1}\right) \cap C^{2}\left(\mathbb{R}^{n} \backslash \overline{B_{1}}\right)
$$

is a radial solution of (11), (2). A direct calculation gives that

$$
D_{i j} u=\left(r u^{\prime \prime}-u^{\prime}\right) \frac{x_{i} x_{j}}{r^{3}}+u^{\prime} \frac{\delta_{i j}}{r}, \quad i, j=1, \cdots, n, \quad r>1,
$$

and the eigenvalues of the Hessian matrix $D^{2} u$ are

$$
\lambda_{1}=u^{\prime \prime}, \lambda_{2}=\cdots=\lambda_{n}=\frac{u^{\prime}}{r} .
$$

From Lemma 1

$$
\gamma=\frac{u^{\prime}}{r} \geq 0, \text { for } r \geq 1
$$

and then $\alpha \geq-1$.

By (1), we have

$$
\begin{gathered}
C_{n-1}^{k-1} u^{\prime \prime}\left(\frac{u^{\prime}}{r}\right)^{k-1}+C_{n-1}^{k}\left(\frac{u^{\prime}}{r}\right)^{k}=1, \\
\left(r^{n-k}\left(u^{\prime}\right)^{k}\right)^{\prime}=\frac{n r^{n-1}}{\mathrm{C}_{n}^{k}} .
\end{gathered}
$$

Then,

$$
\left(u^{\prime}\right)^{k}=\frac{1}{\mathrm{C}_{n}^{k}}\left(r^{k}+\alpha r^{k-n}\right), r>1 .
$$


By Lemma 2 and (14), $u$ is $m$-convex if and only if for $r>1$,

$$
0<\gamma^{k}=\left(\frac{u^{\prime}}{r}\right)^{k}=\frac{1+\alpha r^{-n}}{C_{n}^{k}}<\gamma_{m}^{k},
$$

which is equivalent to

$$
-1 \leq \alpha<+\infty \text {, if } m=k,
$$

and

The lemma is proved.

$$
-1 \leq \alpha \leq \frac{1}{1-\frac{k}{m}}-1=\frac{k}{m-k}, \text { if } m>k .
$$

Lemma 4. Let $n \geq 3$, and $\mu(\alpha)$ be given by (6). Then $\mu(\alpha)$ is strictly increasing on $[-1,+\infty)$ and $\mu(+\infty)=+\infty$.

Proof. Since $s\left[\left(1+\alpha s^{-n}\right)^{1 / k}-1\right]$ is strictly increasing on $\alpha$ in $[-1,+\infty)$ for fixed $s \geq 1$, we have obviously that $\mu(\alpha)$ is strictly increasing on $[-1,+\infty)$.

For $s>|\alpha|^{1 / n}$, it follows from

$$
s\left[\left(1+\frac{\alpha}{s^{n}}\right)^{1 / k}-1\right] \geq \frac{\alpha}{k} s^{1-n}
$$

that

Therefore,

$$
\mu(\alpha) \geq b-\frac{a}{2}+a \int_{1}^{\infty} \frac{\alpha}{k} s^{1-n} \mathrm{~d} s=b-\frac{a}{2}+\frac{a \alpha}{(n-2) k} .
$$

$$
\mu(+\infty)=+\infty
$$

This completes the proof of Lemma 4 .

Now we give the proof of Theorem 2

Proof of Theorem 2. By (14), we have (7), i.e.

$$
u(x)=b+a \int_{1}^{|x|}\left(s^{k}+\alpha \cdot s^{k-n}\right)^{1 / k} \mathrm{~d} s .
$$

Consequently,

$$
\begin{aligned}
u(x)= & b+a \int_{1}^{|x|}\left\{s\left[\left(1+\frac{\alpha}{s^{n}}\right)^{1 / k}-1\right]+s\right\} \mathrm{d} s \\
= & b+\frac{a}{2}\left(|x|^{2}-1\right)+a \int_{1}^{\infty} s\left[\left(1+\frac{\alpha}{s^{n}}\right)^{1 / k}-1\right] \mathrm{d} s \\
& \quad-a \int_{|x|}^{\infty} s\left[\left(1+\frac{\alpha}{s^{n}}\right)^{1 / k}-1\right] \mathrm{d} s \\
= & \frac{a}{2}|x|^{2}+\mu(\alpha)-a \int_{|x|}^{\infty}\left(\frac{\alpha}{k} s^{1-n}+O\left(s^{1-2 n}\right)\right) \mathrm{d} s \\
= & \frac{a}{2}|x|^{2}+\mu(\alpha)+O\left(|x|^{2-n}\right), \quad x \rightarrow \infty,
\end{aligned}
$$

where $\mu(\alpha)$ is given by (6) .

Comparing (15) with (44), we know from Lemma 3 and Lemma 4 that $u$ is $m$ convex if and only if $c \in[\mu(-1),+\infty)$ for $m=k$ and $c \in\left[\mu(-1), \mu\left(\frac{k}{m-k}\right)\right]$ for $m>k$.

The proof of Theorem 2 is completed. 


\section{Proof of Theorem 3}

In this section, we prove Theorem 3 .

Assume that $u(x)=u(r)=u(|x|)$ is the radial solution of (8), (2). The eigenvalues of the Hessian matrix $D^{2} u$ are

$$
\lambda_{1}=u^{\prime \prime}, \lambda_{2}=\frac{u^{\prime}}{r} .
$$

Then, we have

$$
\operatorname{det}\left(D^{2} u\right)=u^{\prime \prime} \cdot \frac{u^{\prime}}{r}=\frac{1}{2 r}\left(\left(u^{\prime}\right)^{2}\right)^{\prime} .
$$

A direct calculation gives that $u^{\prime}>0, u^{\prime \prime}>0$, and

$$
u^{\prime}=\left(r^{2}+d\right)^{1 / 2}
$$

where $d=\left(u^{\prime}(1)\right)^{2}-1$. Therefore,

$$
\begin{aligned}
u=b & +\int_{1}^{|x|}\left(s^{2}+d\right)^{1 / 2} \mathrm{~d} s \\
=b & +\frac{1}{2}\left[|x|\left(|x|^{2}+d\right)^{1 / 2}+d \ln \left(|x|+\left(|x|^{2}+d\right)^{1 / 2}\right)\right] \\
& -\frac{1}{2}\left[(1+d)^{1 / 2}+d \ln \left(1+(1+d)^{1 / 2}\right)\right] .
\end{aligned}
$$

Clearly, the exterior Dirichlet problem (8), (22) has a convex radial solution $u \in$ $C^{1}\left(\mathbb{R}^{2} \backslash B_{1}\right) \cap C^{2}\left(\mathbb{R}^{2} \backslash \overline{B_{1}}\right)$ if and only if $d \geq-1$. Using the formulas

$$
s\left(s^{2}+d\right)^{1 / 2}=s^{2}+\frac{d}{2}+O\left(s^{-2}\right), \quad s \rightarrow+\infty,
$$

and

$$
\ln \left(s+\left(s^{2}+d\right)^{1 / 2}\right)=\ln s+\ln 2+O\left(s^{-2}\right), \quad s \rightarrow+\infty,
$$

we have

$$
u(x)=\frac{1}{2}|x|^{2}+\frac{d}{2} \ln |x|+\nu(d)+O\left(|x|^{-2}\right),
$$

where $\nu(d)$ is given by (9).

Similar to the discussion of the proof of Theorem 2, we know that there exists a unique function $u \in \Phi_{2}$ satisfying (8), (2), (5) if and only if $d \geq-1$ and $c=$ $\mu(d)$.

\section{ACKNOWLEDGEMENTS}

This research was supported by the Program for Changjiang Scholars and Innovative Research Team in University (IRT0908), the National Natural Science Foundation of China (11071020) and the Doctoral Program Foundation of the Institute of Higher Education of China (20100003110003). The authors would like to express deep gratitude to Professor Yanyan Li for his suggestions. 


\section{REFERENCES}

[1] L. A. Caffarelli, Topics in PDEs: The Monge-Ampère equation, Graduate course, Courant Institute, New York University, 1995.

[2] L. A. Caffarelli and Y. Y. Li, An extension to a theorem of Jörgens, Calabi, and Pogorelov, Communications on Pure and Applied Mathematics, 56 (2003), 549-583. MR.1953651 (2004c:35116)

[3] L. A. Caffarelli and Y. Y. Li, Some multi-valued solutions to Monge-Ampère equations, Communications in Analysis and Geometry, 14 (2006), 411-441. MR2260718 (2007i:35067)

[4] L. A. Caffarelli, L. Nirenberg and J. Spruck, The Dirichlet problem for nonlinear secondorder elliptic equations, III. Functions of the eigenvalues of the Hessian, Acta Mathematica, 155 (1985), 261-301. MR806416 (87f:35098)

[5] E. Calabi, Improper affine hyperspheres of convex type and a generalization of a theorem by K. Jörgens, Michigan Math. J., 5 (1958), 105-126. MR0106487 (21:5219)

[6] A. Colesanti and P. Salani, Hessian equations in non-smooth domains, Nonlinear Analysis TMA, 38 (1999), 803-812. MR.1710156 (2001i:35088)

[7] S. Y. Cheng and S. T. Yau, Complete affine hypersurfaces, I. The completeness of affine metrics, Comm. Pure Appl. Math., 39 (1986), 839-866. MR859275 (87k:53127)

[8] K. S. Chou and X. J. Wang, A variational theory of the Hessian equation, Communications on Pure and Applied Mathematics, 54 (2001), 1029-1064. MR.1835381 (2002e:35072)

[9] L. M. Dai and J. G. Bao, On uniqueness and existence of viscosity solutions to Hessian equations in exterior domains, Frontiers of Mathematics in China, 6 (2011), 211-230. MR2780888

[10] P. Delanoë, Partial decay on simple manifolds, Ann. Global Anal. Geom., 10 (1992), 3-61. MR 1172619 (93h:58144)

[11] L. Ferrer, A. Martínez and F. Milán, An extension of a theorem by K. Jörgens and a maximum principle at infinity for parabolic affine spheres, Math. Z., 230 (1999), 471-486. MR 1679973 (2001d:53010)

[12] L. Ferrer, A. Martínez and F. Milán, The space of parabolic affine spheres with fixed compact boundary, Monatsh. Math., 130 (2000), 19-27. MR1762061(2001e:53013)

[13] B. Guan, The Dirichlet problem for a class of fully nonlinear elliptic equations, Communications in Partial Differential Equations, 19 (1994), 399-416. MR1265805 (95c:35100)

[14] K. Jörgens, Über die Lösungen der Differentialgleichung $r t-s^{2}=1$, Math. Ann., 127 (1954), 130-134. MR0062326(15:961e)

[15] J. Jost and Y. L. Xin. Some aspects of the global geometry of entire space-like submanifolds, Results Math., 40 (2001), 233-245. MR1860371 (2002i:53070)

[16] A. V. Pogorelov, On the improper convex affine hyperspheres, Geometriae Dedicata, 1 (1972), 33-46. MR0319126(47:7672)

[17] N. S. Trudinger, On the Dirichlet problem for Hessian equations, Acta Mathematica, 175 (1995), 151-164. MR1368245 (96m:35113)

[18] N. S. Trudinger, Weak solutions of Hessian equations, Communications in Partial Differential Equations, 22 (1997), 1251-1261. MR 1466315 (99a:35077)

[19] N. S. Trudinger and X. J. Wang, Hessian measures. II, Annals of Mathematics (2), 150 (1999), 579-604. MR.1726702(2001f:35141)

[20] N. S. Trudinger and X. J. Wang, The Bernstein problem for affine maximal hypersurfaces, Invent. Math., 140 (2000), 399-422. MR1757001 (2001h:53016)

[21] J. I. E. Urbas, On the existence of nonclassical solutions for two classes of fully nonlinear elliptic equations, Indiana University Mathematics Journal, 39 (1990), 355-382. MR.1089043 (92h:35074)

School of Mathematical Sciences, Beijing Normal University, Laboratory of Mathematics and Complex Systems, Ministry of Education, Beijing 100875, People's Republic of CHINA

School of Mathematical Sciences, Beijing Normal University, Laboratory of Mathematics and Complex Systems, Ministry of Education, Beijing 100875, People's Republic OF CHINA 